\title{
P-0700 : Clinical course of dengue in patients with diabetes mellitus: A Sri Lankan experience
}

${ }^{1}$ Dr. KVC Janaka, ${ }^{1}$ Dr. GMJ Tennakoon, ${ }^{1}$ Dr. CK Jayatilake, ${ }^{1}$ Dr. Hemadri Jayaratne, ${ }^{1}$ Mr. P Muralitharan

${ }^{1}$ Sri Jayewardenepura General Hospital, Sri Lanka.

\section{Introduction}

- Dengue is a major cause of morbidity and mortality throughout tropical and subtropical regions of the world.

- Four distinct dengue viral serotypes, transmitted principally by Aedes aegypti mosquitoes, cause dengue fever (DF) and dengue haemorrhagic fever/dengue shock syndrome (DHF/DSS).

- DF is a self-limited febrile disease. However, the most severe clinical form of dengue (DHF) is defined mostly by the hallmark pathological characteristic of vascular leakage.

- We assessed complications and outcome of dengue patients with diabetes mellitus. Diabetes mellitus is known to exacerbate bacterial infection, but its effect on the severity of viral infection has not been well studied.

\section{Aim}

- This study explored the complications and outcome of dengue patients with diabetes admitted to Sri Jayewardenepura General Hospital, Sri Lanka in year 2018.

\section{Methods}

- Design : Retrospective cross sectional study

- Study settings: Sri Jayewardenepura General Hospital

- Sampling method: Cluster Sampling

- Sample size : 77

- Study Instrument: Objectively designed questionnaire and data derived from medical records

\section{Results}

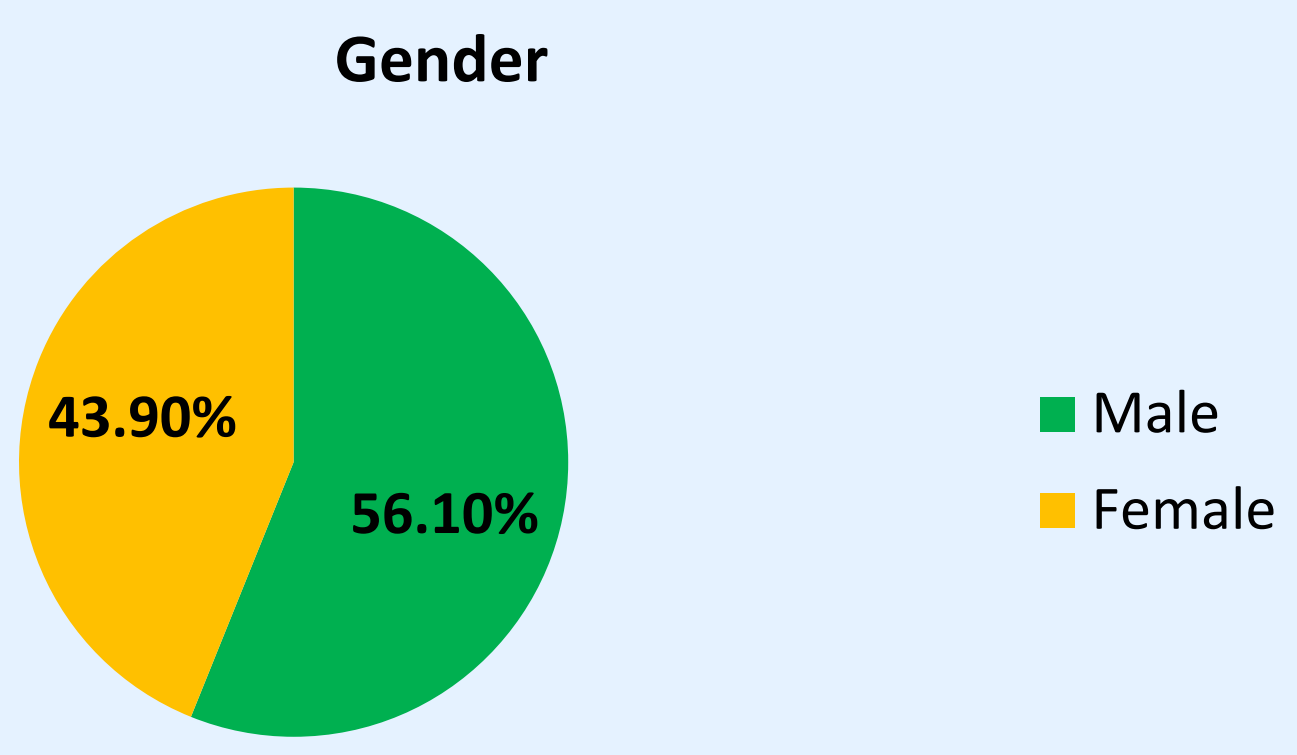

\section{Comorbidities}

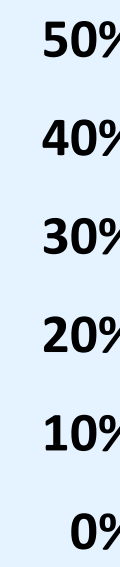

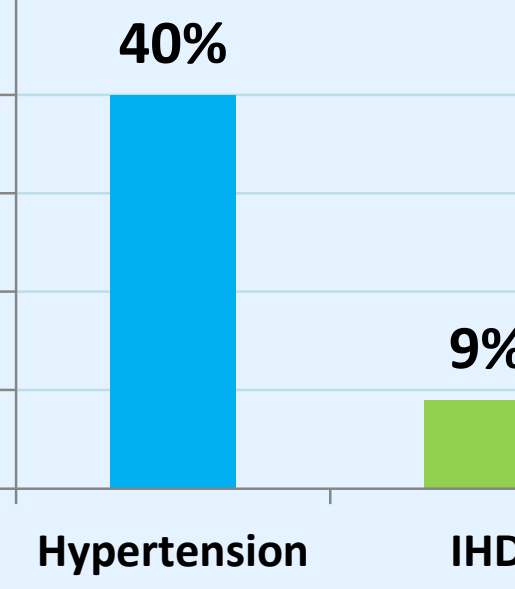

$36.80 \%$

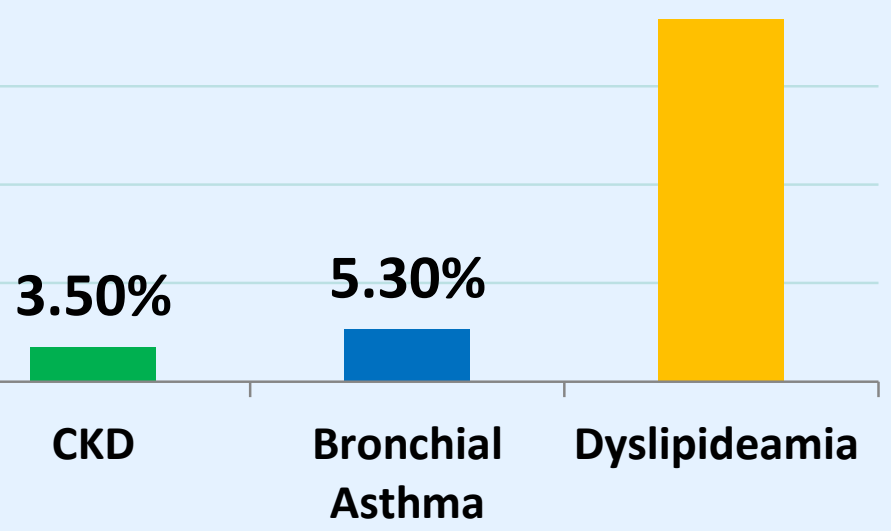

\section{Results Cont.}

Types of dengue fever

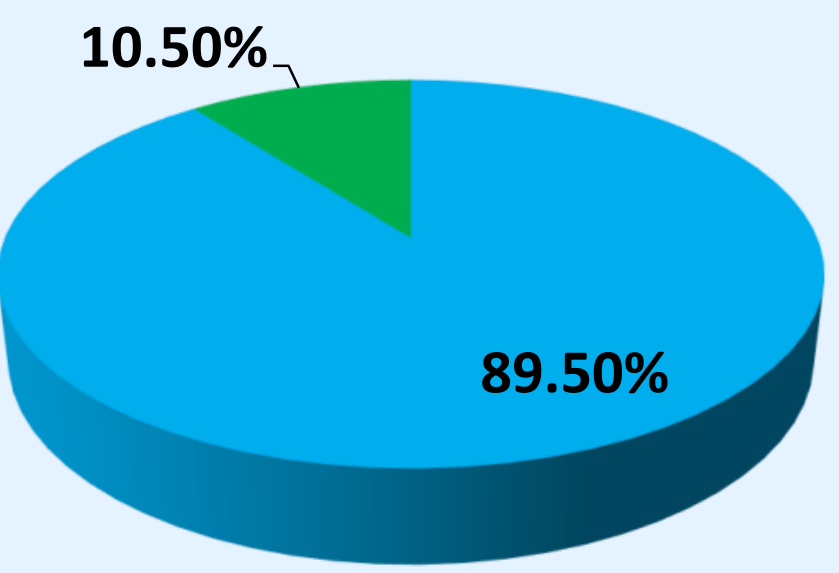

Glycaemic Control

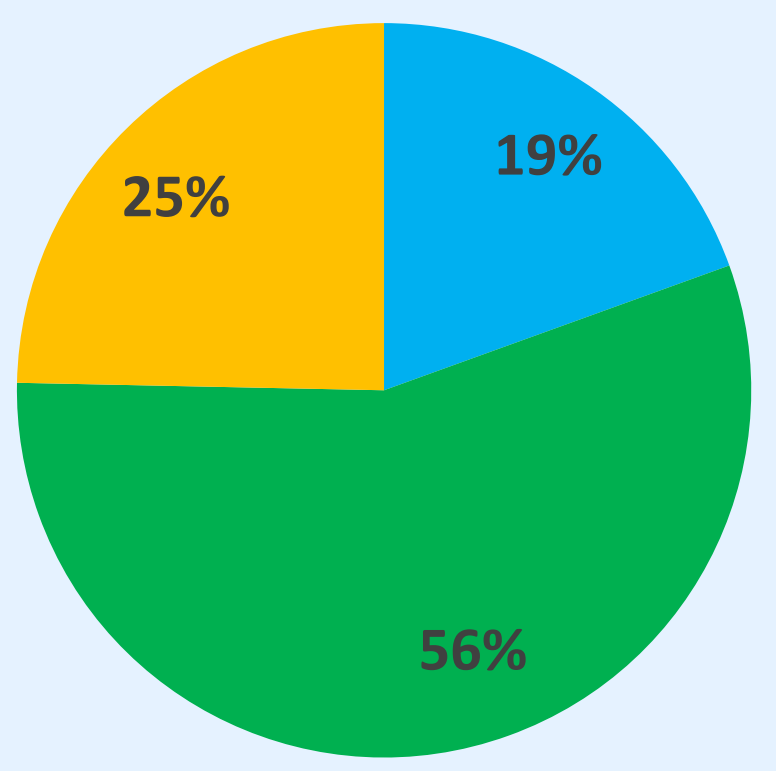

- Good

Average

Poor

Complications

$50 \%$

$40 \%$

$30 \%$

$20 \%$

$10 \%$

$0 \%$

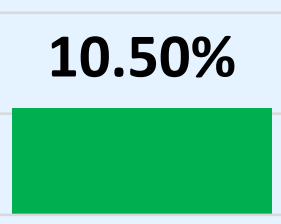

AKI
$41.60 \%$

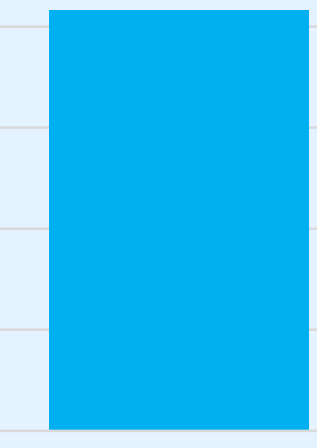

Elevated Liver Enzymes
DF

DHF

\section{Conclusions}

- Presence of diabetes is an additional challenge in patient management due to underlying complications and the effects of blood sugar on fluid status and urine volume.

- DHF or other complications did not show statistically significant difference among patients' glycaemic control.

- Rise of serum creatinine in $10.5 \%$ patients with diabetes who did not have dengue shock syndrome is an important observation.

- This cannot be compared with published other studies as the criteria to diagnose AKI and the overall incidence of shock and mortality varies in the different study populations.

\section{References}

Htun NSN, Odermatt P, Eze IC, Boillat-Blanco N, D'Acremont V, Probst-Hensch N. Is Diabetes a Risk Factor for a Severe Clinical Presentation of Dengue? - Review and Meta-analysis. Halstead SB, ed. PLoS Negl Trop Dis. 2015;9(4):e0003741. doi:10.1371/journal.pntd.0003741

Chen C-Y, Lee M-Y, Lin K-D, et al. Diabetes Mellitus Increases Severity of Thrombocytopenia in Dengue-Infected Patients. Int J Mol Sci. 2015;16(2):3820-3830. doi:10.3390/ijms16023820 